\title{
THE INTRODUCTION OF QUANTUM ELECTRONIC STRUCTURES FOR MEMS DEVICES*
}

\author{
H.L. Hartnagel \\ Institut für Hochfrequenztechnik, Technische Universität Darmstadt, Merckstrasse 25, D-64283 Darmstadt, Deutschland \\ E-mail: hartnagel@hf.tu-darmstadt.de
}

Received June 132005

It is proposed to employ concepts of quantum electronics in microwave MEMS for a number of applications.

Keywords: quantum electronic structures, micro-electro-mechanical structures

PACS: 73.21.Ac

\section{Introduction}

Micro-electro-mechanical structures, MEMS, are now developed with a number of electronic components such as filters and impedance matching circuits, or even whole circuits such a amplifiers and analoguedigital transformers, since the use of a membrane reduces the losses and thus increases the quality factors as required for closely packed multi-channel communication systems [1]. It is notable that there are a number of electronic MEMS circuits where various quantum electronic structures can be usefully incorporated. A typical example is the use of a quantum cascade structure for charge transit effects, such as employed for IMPATT oscillators, or for the recently proposed charge swing for harmonic extraction [2]. In such cases the charge is transferred via a quantum cascade of a series of 2DEG electronic resonators (see Fig. 1) as has been originally introduced for quantum cascade lasers [3]. By bending a MEMS bridge or cantilever, which carries such a succession of quantum resonance structures, it is possible to change the width of the heterostructure resonance valley so that the electronic resonance state occurs at a slightly different energy level. This enables one to affect the electronic charge transfer so that either the current amplitude or the charge transfer time

\footnotetext{
* This publication is dedicated to Professor J. Požela on the occasion of his 80th birthday. He is clearly remembered as someone with an always open mind for new concepts on semiconductor device physics. Therefore this manuscript incorporates a proposal of a new quantum electronic usage for modern devices such as micro-electro-mechanical structures (MEMS), as used for RF circuits.
}

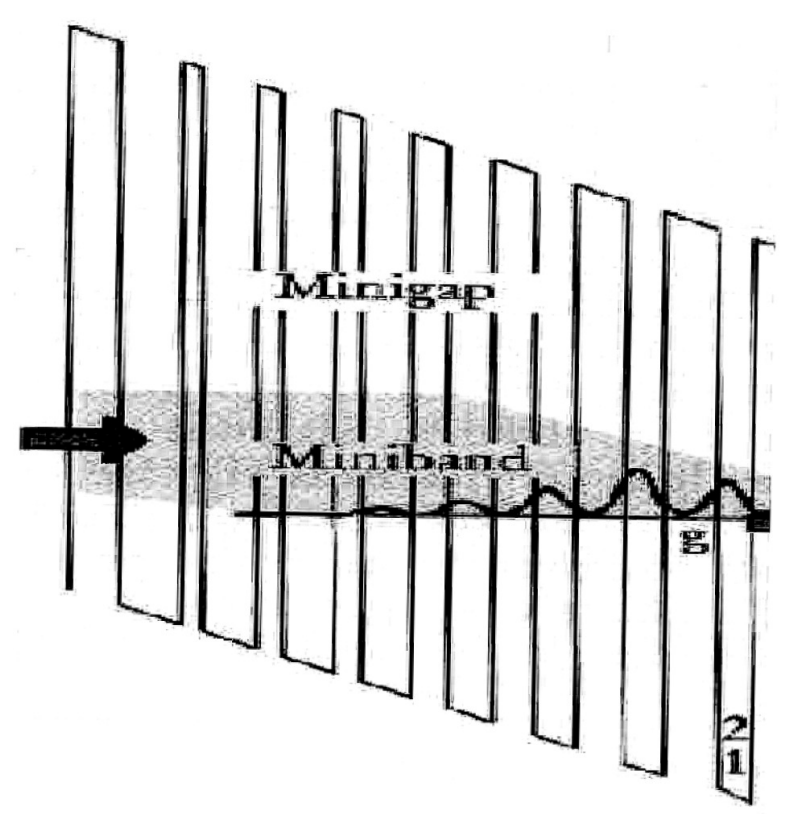

Fig. 1. Each electron resonance level is designed to be at an appropriate level by gap width selection to ensure electron transfer for given applied or internal field. Transfer efficiency and speed is affected by MEMS operation.

is modulated [4]. For a number of MEMS structures with such quantum components involved, this gives the possibility of a fine adjustment of operation.

\section{Material bending affecting quantum structures}

When a cantilever is bent upwards, the material is compressed in the upper part along the cantilever and extended transverse to the cantilever direction. The opposite effects occur in the lower part of the cantilever. 


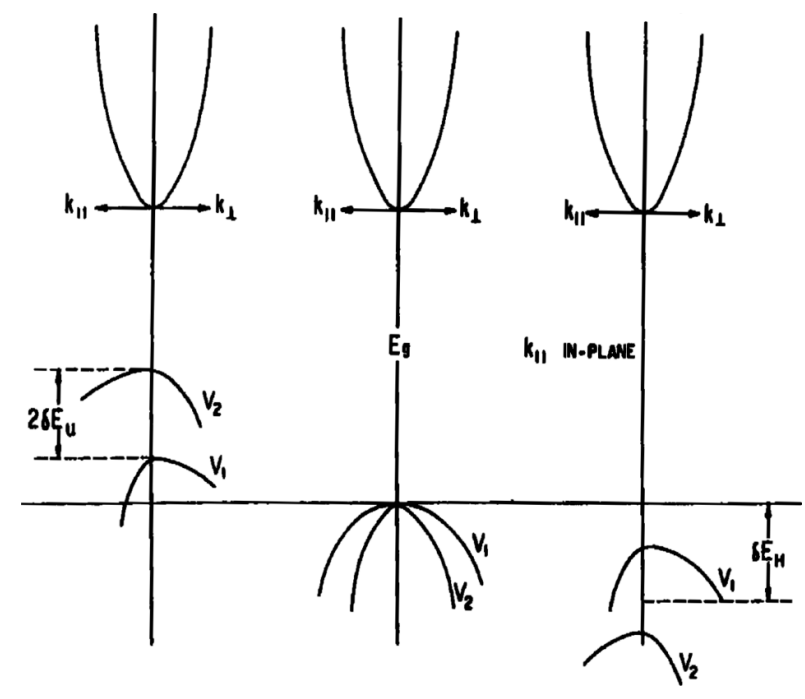

Fig. 2. Effect of strain: band splitting and the removal of degeneracy in heavy and light hole subbands at $k=0$ due to strain caused by lattice mismatch in a hetero-epitaxial system. $\delta E_{\mathrm{H}}$ is a hydrostatic component, $\delta E_{\mathrm{U}}$ is uniaxial component.

If in one of these parts now the quantum structures are introduced by epitaxial growth, they are either compressed or extended. Whereas a cantilever is typically one or two micron thick and several micron wide, the quantum cascade would be 100 nanometre thick only. The change of the resonant valley widths affects the electron wave resonant level. For example, if a layer sequence is epitaxially introduced onto the upper surface of the cantilever so that 2DEG resonant structures are formed as used for quantum cascade lasers, the resonant levels are lowered. Such a lowering then leads to a reduction of the electron current flow when a voltage is applied across the cascade structure. An estimate of the material displacement can lead to an extension of around 5\%, and correspondingly the resonance level is

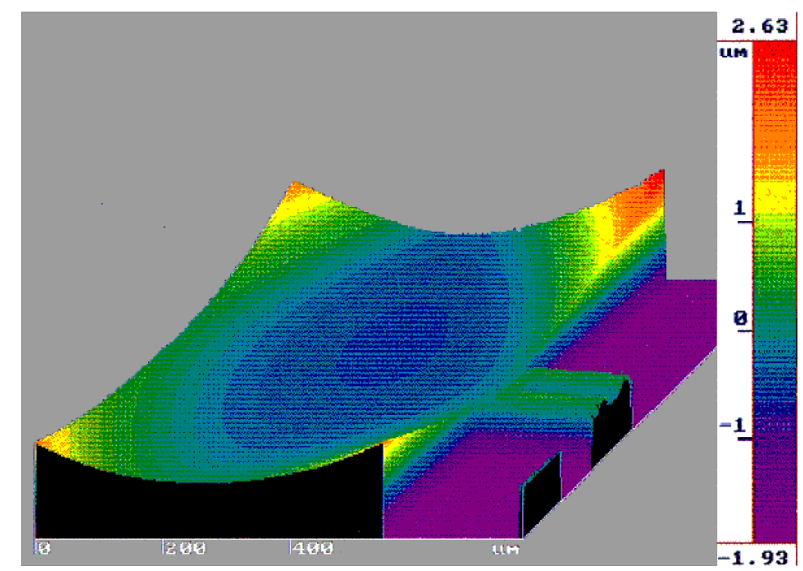

changed by roughly $5 \%$. This can in fact lead even to a complete interruption of current.

There are also other effects possible to change a current flow. For example, the valence band details are strongly affected by compression or extension of the semiconducting material (Fig. 2) so that a current across quantum wells can be interrupted in $p$-type material.

It is quite easily possible to design hetero-epitaxial sandwiches with small lattice mismatches so that inbuilt strains can enhance or orientate the modulation of the quantum well widths in the desired manner (see Fig. 3). Small mismatches can be easily grown epitaxially.

If for example a mismatched layer is grown under the quantum well cascade so that it is compressed without bending the MEMS cantilever (i. e. the lattice constant of an intermediate layer is larger than that of the cascade sandwich), the bending upwards will widen the quantum wells over a larger bending radius.

There exists a large range of design possibilities with such internal mismatch strain.

\section{Discussion of device applications}

Such a current modulation by the bending of a cantilever or other MEMS structures can be used for a number of components. First, there are acoustic resonators for time keeping [5] such as watches or mobile communication units, where the motion of a mass is identified for example by capacitive or inductive fields. If the support bridge, acting as a spring, carries now a quantum cascade current, this can be modulated by the motion of the resonating mass structure.

Another application would be an acceleration sensor, where again a mass structure is affected by

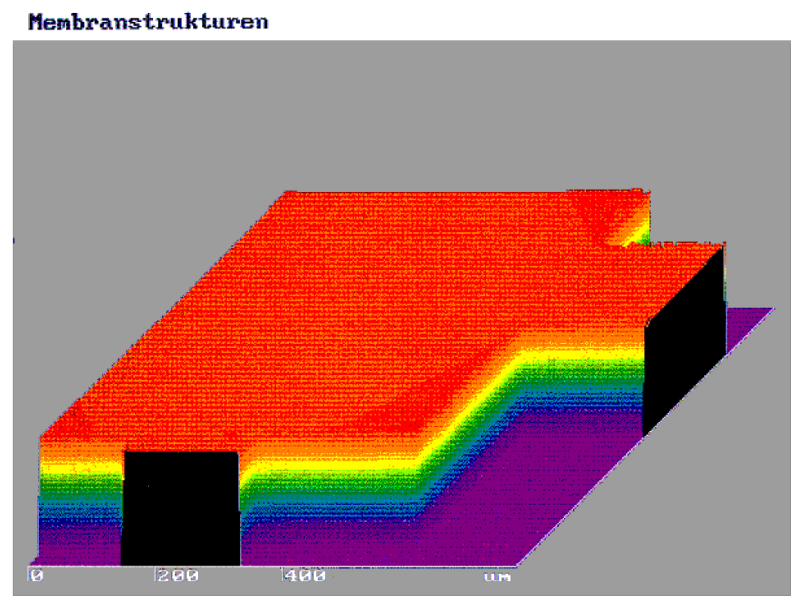

Fig. 3. Demonstration of inbuilt strain by bending of a MEMS surface. 
acceleration as experienced in motor cars when a crash needs to lead to a release of the air bags. So far this has also been made usually capacitively.

A third application would be the signal current of a filter going across a quantum cascade, which represents a MEMS-adjustable resistance. The resonance frequency can thus be slightly changed by the MEMSmodified current flow. In this way fine tuning of filter performance can be achieved.

There are a number of further device possibilities where such modifications of quantum cascade currents can be used, where quantum cascading could be made of 2DEG, 1DEG, or ODEG structures with good coupling. The tunnelling effect gives also slightly different charge transit times, depending on the tunnelling distance. Again, this can be useful for various electronic applications.

\section{Conclusions}

By using quantum cascade concepts for those parts of a MEMS where material is strongly compressed or extended, the cascade current can be modulated then.
This enables one to consider a number of interesting applications in the field of RF MEMS.

\section{References}

[1] H.L. Hartnagel, Millimeter-wave and terahertz devices based on MEMS concepts, in : Proceedings of GAAS, European Microwave Week 2002, 23-27 September 2002, Milan, Italy, 249-252.

[2] H.L. Hartnagel, Proposal of a new type of non-linear component for harmonic extraction, Frequenz 53, 5-6 (1999).

[3] J. Faist, F. Capasso, D.L. Sivco, C. Sirtori, A.L. Hutchinson, and A.Y. Cho, Quantum cascade laser, Science 264, 553-556 (1994).

[4] H.L. Hartnagel and M. Dragoman, Modulation of quantum-electronic devices by bending of special MEMS structure, invited paper at the International Conference CAS, Sinaia, Romania, October 2004.

[5] K. Hjort, G. Schweeger, A. Dehé, K. Fricke, and H.L. Hartnagel, Thickness-field excited thickness-shear resonators in (110) GaAs, Appl. Phys. Lett. 66, 326-328 (1995).

\title{
KVANTINĖS ELEKTRONIKOS DARINIU ITERPIMAS I MEMS PRIETAISUS
}

\author{
H.L. Hartnagel \\ Darmštato technikos universitetas, Darmštatas, Vokietija
}

\section{Santrauka}

Mikroelektromechaninių darinių prietaisuose siūloma panaudoti kvantinės elektronikos darinius, nurodomos tokių prietaisų pritaikymo galimybès. 